\title{
Fan the flame: trazodone-induced mania in a unipolar depressed patient with stable sertraline treatment
}

This article was published in the following Dove Press journal:

Neuropsychiatric Disease and Treatment

24 August 2017

Number of times this article has been viewed

\author{
Jianbo $\mathrm{Hu}^{1,2, *}$ \\ Jianbo Lai ${ }^{1,2, *}$ \\ Hanzhi Zheng ${ }^{3}$ \\ Shaohua $\mathrm{Hu}^{1,2}$ \\ Yi Xu ${ }^{1,2}$
}

'Department of Psychiatry, First Affiliated Hospital, Zhejiang University

School of Medicine, Hangzhou,

China; ${ }^{2}$ The Key Laboratory of

Mental Disorder's Management in

Zhejiang Province, Hangzhou, China;

${ }^{3}$ Department of Clinical Medicine,

Zhejiang University School of

Medicine, Hangzhou, China

*These authors contributed equally to this work
Correspondence: Shaohua Hu

Department of Psychiatry, the First Affiliated Hospital, Zhejiang University

School of Medicine, 79 Qingchun Road,

Hangzhou 310003, China

Tel +86 57I 56723002

Fax +86 57| 5672300 I

Email dorhushaohua@zju.edu.cn

YiXu

Department of Psychiatry, the First Affiliated Hospital, Zhejiang University

School of Medicine, 79 Qingchun Road,

Hangzhou 310003, China

Tel +86 I380574 6579

Fax +86 57I 5672300 I

Email xuyizju@zju.edu.cn
Abstract: Depressed patients often complain of sleep disturbance. Routine antidepressive strategies sometimes fail to deal with this intractable issue. Indeed, the supplementation of sleep promoting antidepressants (eg, trazodone, mirtazapine, and agomelatine) is prevalent in clinical practice. However, the combination of different antidepressants may increase the affective lability. Herein, we document a patient with unipolar depression who was compliant with sertraline treatment and who dramatically switched to mania after adding trazodone as a sleeping aid. This case extended our understanding of the potential manic-switching risk when trazodone is used to promote sleep.

Keywords: trazodone, sertraline, depression, mania

\section{Introduction}

Difficulty in initiating or maintaining sleep is not uncommon among patients with affective disorders. ${ }^{1}$ Comorbid insomnia is associated with severe psychiatric symptoms and significantly impairs the quality of life. ${ }^{1,2}$ Nevertheless, typical antidepressants (eg, selective serotonin reuptake inhibitors and serotonin-norepinephrine reuptake inhibitors) can help to improve the mood, but sometimes fail to handle the sleep problem properly. ${ }^{1,3}$

As was shown in a recent large-scale survey, off-label use of trazodone for insomnia accounted for nearly a quarter of all off-label antidepressant use. ${ }^{4}$ However, the potential risk associated with off-label trazodone use received limited attention. Furthermore, due to weak evidence of the outcome and appropriateness, the use of trazodone for sleep onset and maintenance difficulties in adult patients was not recommended by the recently updated guidelines of American Academy of Sleep Medicine. ${ }^{5}$

According to published data, common adverse events of trazodone are gastrointestinal, cardiovascular, and neurologic effects. ${ }^{6}$ As an antidepressant approved by the US Food and Drug Administration, the risk of trazodone inducing manic-switching was relatively low in patients with bipolar disorder when coprescribed with mood stabilizers. ${ }^{7}$ To date, there are sparse cases of trazodone-induced mania reported, mostly in patients with predisposing factors. ${ }^{7}$ Herein, we present a middle-aged male patient with unipolar depression who was in remission with compliance to sertraline treatment and switched to mania after adding trazodone to promote sleep.

\section{Case presentation}

A 36-year-old male patient was taken to our hospital by his family members because of elevated mood and aggressive behaviors. His medical history was collected by 
detailed inquiry. In his early adolescence, the patient experienced his first episode of depression, which was ascribed to the bad relationship between his parents. Since then, he complained that his mood had swung frequently, with declined interest in daily activities and loss of appetite. This patient condemned himself as solitary, endocentric, and cowardly. He had been a Christian for approximately 10 years. After graduating from university, he worked as a physics teacher in a local high school. His job performance at school was proficient. Not until this patient heard of health education on mental illnesses did he first come to a local clinic for psychiatric counseling (nearly 8 months prior to admission). On that occasion, he complained of feeling upset, irritable, and fatigued. He was diagnosed with generalized anxiety disorder and was prescribed tandospirone and propranolol. Nonetheless, these regimens were discontinued by the patient after 2 weeks because of the unsatisfactory outcome. He then visited our hospital and the psychiatrist revised the diagnosis to major depressive disorder. He was given sertraline at a dose of $100 \mathrm{mg} /$ day. About 2 months after initiating sertraline treatment, this patient still felt depressed and was not interested in daily activities. The dose of sertraline was, therefore, increased into $150 \mathrm{mg} /$ day. From then on, our patient had felt at ease with sertraline treatment for nearly 4 months. His performance at work and interpersonal relationships also improved.

About 1 week prior to admission, the patient revisited our hospital and complained of waking up early in the morning, however his emotional state was stable. Trazodone at a dose of $25 \mathrm{mg} /$ night was added to promote sleep. He felt extremely excited after taking trazodone for the first time; he slept only 2 hours that night, and became talkative and conceited. In the following 2 days, he continued to take trazodone, however on day 3 he discontinued trazodone due to abnormal behavior. He preached Christianity during his class which was forbidden according to the school disciplines. He even sent emails to the White House to seek protection. Also, our patient professed that he was conferred superpowers by God. The day before admission, the patient felt that he was possessed by a supernatural spirit and was told to save the world before the coming doomsday. He abused his superiors at a public occasion because of their insincerity. Consequently, he was sent to our hospital for management.

On admission, the patient could not stop talking and asserted the ability to understand the inner world of others. An extensive physical examination revealed no neurological deficits. No substance abuse or toxin exposure was reported. Laboratory screenings, including routine analysis of blood, urine, stool, biochemistry, thyroid hormones, coagulation function, C-reactive protein, cortisol, adrenocorticotropic hormone, tumor and infectious biomarkers, were all within the normal limits. Cranial magnetic resonance scanning was also normal. The patient was diagnosed with bipolar I disorder, manic episode, according to the Diagnostic and Statistical Manual of Mental Disorders, 5th edition. Quetiapine was gradually titrated to $700 \mathrm{mg}$ per night over 1 week accompanied with $5 \mathrm{mg}$ of haloperidol twice a day for 5 consecutive days. His emotional state gradually stabilized after about 2 weeks of hospitalization. After discharging from hospital, the patient took quetiapine $700 \mathrm{mg}$ every night and remained euthymic during outpatient follow-ups.

\section{Ethics}

This case study obtained approval from the Institute Ethical Committee of the First Affiliated Hospital, Zhejiang University School of Medicine. The patient and his family members signed informed consent to publish case details.

\section{Discussion}

We present an interesting case in which a small dose of trazodone augmentation to sertraline treatment dramatically induced mood-switching. Of note, trazodone was used as a hypnotic agent in our patient. Given its massive use in the general population, this case study is of practical importance when coprescribing trazodone with other antidepressants.

As a serotonin receptor antagonist and serotonin reuptake inhibitor (SARI), trazodone was classically used as an antidepressant agent. Nevertheless, off-label use of trazodone is commonly found in patients with various medical conditions, including anxiety disorder, obsessive-compulsive disorder, substance use disorder, eating disorder, sexual dysfunction, and certain pain conditions. ${ }^{6}$ Besides, trazodone is often used to cope with insomnia in clinical practice. ${ }^{4,8}$ Two mechanisms may underlie the sleep-enhancing effects of trazodone, including the blockage of serotonergic 5- $\mathrm{HT}_{2 \mathrm{~A}}$ receptors and histamine $H_{1}$ receptors. ${ }^{9}$ It is noteworthy that off-label use of trazodone should be cautiously evaluated on the basis of individual differences.

To date, less than 20 cases of manic-switching associated with trazodone treatment have been documented. ${ }^{7}$ According to the literature review, these patients were diagnosed with either unipolar depression or bipolar disorder. ${ }^{7}$ The daily dose of trazodone ranged from $50 \mathrm{mg}$ to $400 \mathrm{mg}^{7}$ It was interesting that these patients were treated with trazodone monotherapy or in combination with other antidepressants, and none of them were coadministered with mood stabilizers. The onset of manic-switching time following trazodone treatment varied from 4 days to 4 weeks. ${ }^{7}$ Of note, these mania 
events can be well-controlled with cessation or reduction of trazodone, administration of haloperidol, or adding mood stabilizers. ${ }^{7}$ As for our patient, there were three main differences from the aforementioned reports, including the low dose of trazodone ( $25 \mathrm{mg}$ per night), the rapid onset of mania (the first night of taking trazodone), and the coprescribed antidepressant sertraline (none of the previous cases were associated with sertraline).

In the view of pharmacokinetics, the in vivo metabolism of trazodone was predominantly via CYP3A4 enzyme and relatively less via CYP2D6 enzyme. ${ }^{10,11}$ Notably, these two cytochrome 450 isotypes were also involved in the metabolism of sertraline. ${ }^{12}$ Therefore, addition of trazodone as a sleeping aid may increase the serum concentration of sertraline, and further interfere with the balance of neurotransmitter systems. However, other explanations for the manic behaviors in our patient should also be taken into consideration. It has been reported that almost $8 \%$ of patients with a diagnosis of unipolar depression may encounter moodswitching when taking antidepressants. ${ }^{13}$ For our patient, some manifestations prior to the manic episode, such as mood instability and irritability, may indicate an unrecognized or potential condition of bipolar disorder. We should pay attention to the new-onset insomnia prior to the manic episode. The symptom of insomnia could be secondary to depression, or a trigger of manic episode, or even an initial manifestation of an evolving manic episode. Therefore, clinicians should carefully evaluate every symptom in depressed patients, especially the risk factors for bipolar disorder, before formulating the pharmacological strategy. Moreover, although manic-switching was an unwanted adverse effect to our patient, an important lesson can be learned that trazodone can be used to augment the efficacy of other antidepressants, especially in patients with sleep problems. This perspective was in accordance with another case study, in which addition of trazodone as a third antidepressant to paroxetine and mirtazapine successfully eased the symptoms of depressed mood and insomnia in a patient with treatment-resistant depression. ${ }^{14}$ However, safety, tolerance, and efficacy of adding trazodone to other antidepressants should be observed in future investigations.

\section{Conclusion}

We present a scenario of trazodone-induced mania in a depressed patient with compliance to sertraline treatment. This case indicated that when using trazodone as a hypnotic agent close monitoring was necessary to evaluate the potential risk of manic-switching, especially in the early stage of therapy.

\section{Acknowledgments}

This work was supported by grants of the Medicine and Health Program of Zhejiang Province (2014ZDA008, 2013RCA017, and 2015KYB136), the National Key Basic Research Program (2016YFC1307104), the Public Welfare Project of Science Technology Department of Zhejiang Province (2015C33133), the National Clinical Research Center for Mental Health Disorders (2015BAI13B02), the Key Research Project of Zhejiang Province (2015C03040), and the Research Program of Education Department in Zhejiang Province (Y201430870). We acknowledge Dr Pornkanok Prukpitikul for helping to polish the language.

\section{Disclosure}

The authors report no conflicts of interest in this work.

\section{References}

1. Bradley AJ, Webb-Mitchell R, Hazu A, et al. Sleep and circadian rhythm disturbance in bipolar disorder. Psychol Med. 2017;47(9): 1678-1689.

2. Monti JM. The effect of second-generation antipsychotic drugs on sleep parameters in patients with unipolar or bipolar disorder. Sleep Med. 2016;23:89-96.

3. Rumble ME, White KH, Benca RM. Sleep Disturbances in Mood Disorders. Psychiatr Clin North Am. 2015;38(4):743-759.

4. Wong J, Motulsky A, Abrahamowicz M, Eguale T, Buckeridge DL, Tamblyn R. Off-label indications for antidepressants in primary care: descriptive study of prescriptions from an indication based electronic prescribing system. BMJ. 2017;356:j603.

5. Sateia MJ, Buysse DJ, Krystal AD, Neubauer DN, Heald JL. Clinical Practice Guideline for the Pharmacologic Treatment of Chronic Insomnia in Adults: An American Academy of Sleep Medicine Clinical Practice Guideline. J Clin Sleep Med. 2017;13(2):307-349.

6. Khouzam HR. A review of trazodone use in psychiatric and medical conditions. Postgrad Med. 2017;129(1):140-148.

7. Wichniak A, Jarkiewicz M, Okruszek Ł, Wierzbicka A, Holka-Pokorska J, Rybakowski JK. Low risk for switch to mania during treatment with sleep promoting antidepressants. Pharmacopsychiatry. 2015;48(3): 83-88.

8. Giannaccini G, Masala I, Palego L, et al. Melatonin and pro-hypnotic effectiveness of the antidepressant Trazodone: A preliminary evaluation in insomniac mood-disorder patients. Clin Biochem. 2016;49(15): 1152-1158.

9. Wichniak A, Wierzbicka A, Jernajczyk W. Sleep and antidepressant treatment. Curr Pharm Des. 2012;18(36):5802-5817.

10. Rotzinger S, Fang J, Baker GB. Trazodone is metabolized to m-chlorophenylpiperazine by CYP3A4 from human sources. Drug Metab Dispos. 1998;26(6):572-575.

11. Rotzinger S, Fang J, Coutts RT, Baker GB. Human CYP2D6 and metabolism of m-chlorophenylpiperazine. Biol Psychiatry. 1998;44(11): 1185-1191.

12. Obach RS, Cox LM, Tremaine LM. Sertraline is metabolized by multiple cytochrome P450 enzymes, monoamine oxidases, and glucuronyl transferases in human: an in vitro study. Drug Metab Dispos. 2005;33(2): 262-270.

13. Baldessarini RJ, Faedda GL, Offidani E, et al. Antidepressant-associated mood-switching and transition from unipolar major depression to bipolar disorder: a review. J Affect Disord. 2013;148(1):129-135.

14. Lopes R, Alves JC, Rego RG. Trazodone addition to paroxetine and mirtazapine in a patient with treatment-resistant depression: The pros and cons of combining three antidepressants. Case Rep Med. 2016;2016: article 5362485 . 


\section{Publish your work in this journal}

Neuropsychiatric Disease and Treatment is an international, peerreviewed journal of clinical therapeutics and pharmacology focusing on concise rapid reporting of clinical or pre-clinical studies on a range of neuropsychiatric and neurological disorders. This journal is indexed on PubMed Central, the 'PsycINFO' database and CAS,

and is the official journal of The International Neuropsychiatric Association (INA). The manuscript management system is completely online and includes a very quick and fair peer-review system, which is all easy to use. Visit http://www.dovepress.com/testimonials.php to read real quotes from published authors.

Submit your manuscript here: http://www.dovepress.com/neuropsychiatric-disease-and-treatment-journal 